\title{
Synthesis, Characterization and Antimicrobial Studies of Schiff Base Ligand-Derived from Amoxicillin and Benzaldehyde
}

\author{
Asif Iqbal and Farhana Hoque \\ Department of Pharmacy, University of Science and Technology Chittagong (USTC), Chittagong-1079, Bangladesh
}

Received: June 17, 2016; Accepted: July 21, 2016; Published (Web): July 31, 2016

\begin{abstract}
Advanced research into synthetic chemistry has pointed out several organic compounds with antimicrobial potential. Schiff bases are one of such compounds. This research aims at the synthesis of a Schiff base out of a fairly common antibiotic amoxicillin and benzaldehyde. The synthesized compound was characterized by different spectroscopic techniques. It was also subjected to melting point determination, TLC and solubility tests. The Schiff base was screened for antibacterial and antifungal activities in-vitro by disc diffusion method.
\end{abstract}

Key words: Schiff base, amoxicillin, benzaldehyde, antibacterial, antifungal, MIC

\section{Introduction}

Infectious diseases have been a major health problem for decades, especially in the developing and underdeveloped countries. Moreover, the tendency of microorganisms to develop resistance to antibiotics further necessitates the urge to develop newer drugs.

Schiff bases are an important class of organic compounds, synthesized from the condensation of primary amines with carbonyl groups (Arulmurugan et al., 2010). They were first reported by Hugo Schiff in 1864 (Schiff, 1864). Structurally a Schiff base is a nitrogen analogue of a carbonyl compound in which the carbonyl group is replaced by an imine or azomethine group (Dhar et al., 1982). The imine group in these compounds is proved to be responsible for various biological activities, antibacterial and antifungal being prominent (Przybylski et al., 2009). The general formula of a Schiff base is $\mathrm{RHC}=\mathrm{N}-\mathrm{R}_{1}$, where $\mathrm{R}$ and $\mathrm{R}_{1}$ are alkyl, aryl, cycloalkyl or heterocyclic groups (Arulmurugan et al., 2010).

Amoxicillin, a $\beta$-lactam antibiotic belonging to the class of penicillins, is an acid stable semi-synthetic drug shown to be effective against a wide range of infections caused by numerous Gram-positive and Gram-negative strains of bacteria both in humans and animals (Brogden et al., 1979, El-Sooud et al., 2004). Originally introduced in the early 1970s for oral use in the U.K, it has gradually established itself as an effective remedy to a wide range of infectious diseases (Gordon et al., 1972). Amoxicillin monograph is available in the United States, British and Indian Pharmacopeias (USP 2007, BP 2009, IP 2007). It is also on the World Health Organization's list of Essential Drugs (WHO 2013).

Considering these facts, a Schiff base was synthesized from amoxicillin and benzaldehyde and its antimicrobial properties was evaluated to establish it as an effective therapeutic agent.

\section{Materials and Methods}

Chemicals and reagents: All chemicals were of analytical grade and used as received. All solvents were purified using conventional methods.

Physical measurements: Infrared spectrum was acquired on a Shimadzu Infrared Spectrophotometer using potassium bromide disc, within the range of $4000-500 \mathrm{~cm}^{-1}$ at the University of Chittagong. UVvisible spectrum was recorded on a UV-visible spectrophotometer at the University of Science and

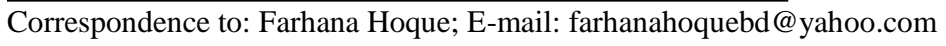


Technology Chittagong (USTC). ${ }^{1} \mathrm{H}$ NMR spectrum was obtained on a Bruker NMR spectrometer using deutarated methanol as solvent, at BCSIR laboratories, Dhaka. Melting point was determined on an electrothermal melting point apparatus and TLC was performed using conventional TLC plates at the University of Science and Technology Chittagong (USTC).

General method of Schiff base preparation: Amoxicillin $12 \mathrm{mmol}$ and $12 \mathrm{mmol}$ of benzaldehyde were taken in a round bottom flask along with methanol as solvent. The magnet was introduced into the reaction medium and the flask was placed in a large beaker containing soya bean oil, standing on the hot plate of the magnetic stirrer. The condenser was fitted along with a thermometer. The machine was switched on and the reading on the thermometer was noted until it reached $80{ }^{\circ} \mathrm{C}$. After the desired temperature was reached, the mixture was refluxed for 24 hours. Then the apparatus was switched off and the reaction mixture was cooled in an ice water bath and left for 24 hours in the refrigerator to allow condensation of the product. After the product was condensed, it was filtered off and collected. The remaining reaction mixture was collected in a beaker which was placed on the hot plate magnetic stirrer to evaporate the remaining solvent. After solvent evaporation, the beaker was placed in an ice water bath to crystallize the remaining product which was then filtered. This along with the previous batch of filtrate was dissolved in methanol. The methanolic solution of the product was placed in an ice water bath to complete re-crystallization. The re-crystallization process was repeated 3 times until pure product was obtained. The product was dried in a dessicator over anhydrous silica gel.

Antimicrobial screening: Four pathogenic bacteria viz., Salmonella typhi (Gram negative), Escherichia coli (Gram negative), Staphylococcus aureus (Gram positive), Bacillus subtilis (Gram positive) and two species of fungus Candida albicans and Aspergillus fumigatus were collected from the Department of Microbiology, University of Chittagong. Nutrient agar was used as a culture media for bacteria while potato dextrose agar was employed in antifungal studies. The Schiff base was dissolved in methanol at a concentration of $0.030 \mathrm{mg} / \mathrm{ml}$. The in vitro antimicrobial activity of the Schiff base was assessed by disc diffusion method. The diameter of the zone of inhibition produced by the compound was compared with the parent drug, amoxicillin.

\section{Results and Discussion}

Infrared spectroscopy: The results of infrared spectral analysis are presented in table 1 . The spectrum displayed characteristic bands for the phenolic $\mathrm{OH}$ group at $3425 \mathrm{~cm}^{-1}$ and $3410 \mathrm{~cm}^{-1}$, a pair of bands for aromatic $\mathrm{C}=\mathrm{C}$ at $1512 \mathrm{~cm}^{-1}$ and $1431 \mathrm{~cm}^{-1}$ and most importantly for an azomethine group $\mathrm{C}=\mathrm{N}$ at $1604 \mathrm{~cm}^{-1}$.

Table 1. Infrared spectral data of the synthesized compound.

\begin{tabular}{ccccc}
\hline \multicolumn{5}{c}{ Assignment $\left(\mathrm{cm}^{-1}\right)$} \\
\hline Azomethine $\mathrm{C}=\mathrm{N}$ & Amide $\mathrm{C}=\mathrm{O}$ & Ar-C=C & Ar-OH & Ar-H \\
\hline $1604(\mathrm{~s})$ & 1651 (strong) & 1512 and 1431 (medium) & 3425 and 3410 (strong) & 3010 (weak) \\
\hline
\end{tabular}

$U V$-visible spectroscopy: The electronic absorption spectra of the Schiff base was recorded at room temperature using distilled water as solvent. The wavelength maximum was observed at $370.5 \mathrm{~nm}$.

${ }^{l} H$ NMR spectroscopy: The observed chemical shifts for the different types of protons in the ${ }^{1} \mathrm{H}$ NMR spectrum are presented in table 2. The ${ }^{1} \mathrm{H}$ NMR spectrum of the prepared compound indicated a singlet at $8.1 \mathrm{ppm}$ owing to the presence of an azomethine proton $(\mathrm{CH}=\mathrm{N})$. Another significant observation was the presence of aryl protons over a range of $6.8-7.6$ ppm occuring as multiplets. A singlet for a hydroxyl group was observed at $3.8 \mathrm{ppm}$, while a secondary amine proton was identified at $4.5 \mathrm{ppm}$. It has been confirmed through research that azomethines show chemical shifts in the vicinity of $8-8.7$ (Tai et al., 2007). 
Table 2. ${ }^{1}$ H NMR spectral data of the synthesized compound.

\begin{tabular}{cc}
\hline $\begin{array}{c}\text { Chemical shift } \\
\text { (in ppm) }\end{array}$ & Assignment of protons \\
\hline 1.2 & singlet, Methyl proton \\
$6.8-7.6$ & multiplet, Phenyl protons \\
8.1 & singlet, $\mathrm{CH}=\mathrm{N}$ - proton \\
3.8 & singlet, hydroxyl proton \\
\hline
\end{tabular}

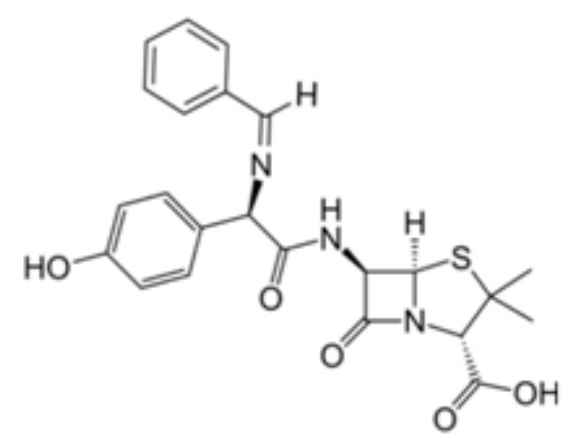

Figure 1. Possible structure of the Schiff base ligand-derived from amoxicillin.

Melting point determination: The synthesized Schiff base demonstrated a characteristic melting point within the range of $179-180^{\circ} \mathrm{C}$.
$R_{f}$ value determination: A mixture of the solvents $\mathrm{N}$-hexane and methanol was used at a ratio of 10:4 (Nhexane: methanol). The $\mathrm{R}_{\mathrm{f}}$ value of 0.33 was observed for the Schiff base while the parent drug amoxicillin demonstrated a value of 0.20 .

Antibacterial activity: The target compound was screened for antibacterial activity against two species of Gram-positive bacteria (Staphylococcus aureus, Bacillus subtilis) and two species of Gram-negative bacteria (Salmonella typhi, Escherichia coli). Results of the disc diffusion studies are depicted in figure 2. It was observed that the Schiff base was more active against Gram-negative strains than Gram-positive. However, in most cases, the parent drug was more active than the prototype. Diameters of the zone of inhibition (in $\mathrm{mm}$ ) for the prototype compound in case of Stap. aureus, $B$. subtilis, $S$. typhi and E. coli were found to be 13, 11, 25 and $12 \mathrm{~mm}$, respectively. Results for the same selection of bacteria in case of the standard drug amoxicillin were 30, 21, 18 and $13 \mathrm{~mm}$, respectively. While the compound appeared to be more active against $S$. typhi (Table 3) than its precursor, results on Stap. aureus can be deemed satisfactory and the small variations for E.coli can be neglected.

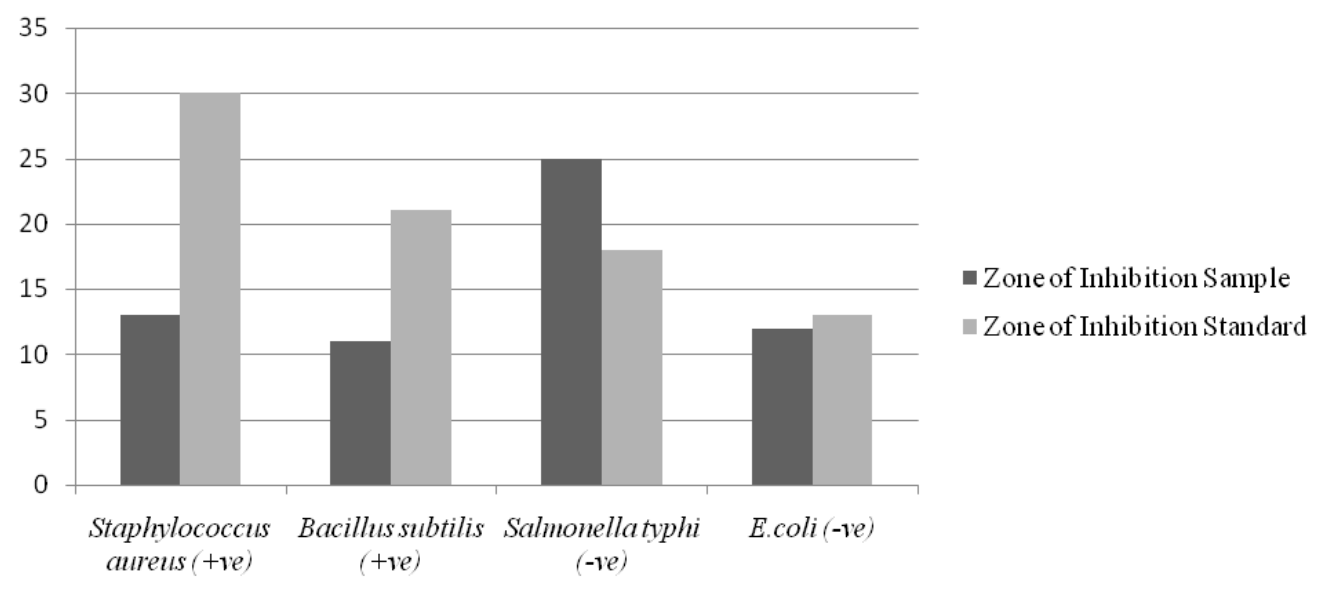

Figure 2. Comparison of zone of inhibition of the Schiff base and standard.

Antifungal activity: Antifungal studies were carried out by disc diffusion technique on potato dextrose agar against Candida albicans and Aspergillus fumigatus. Unfortunately, the compound showed no activity, so the results were not compared.

\section{Conclusion}

Here, we developed a convenient method for the synthesis of Schiff Base ligand from amoxicillin and benzaldehyde using condensation reaction. This ligand has been characterized on the basis of IR, ${ }^{1} \mathrm{H}-\mathrm{NMR}$ and 
UV spectral data. Antimicrobial activity was observed against E. coli, S. typhi, B. rabtilis, S. aureus, $C$. albicans and A. fumigatus. The ligand was found to be more active than the standard against $S$. typhi. It also showed good activity against the other three bacteria. But it failed to show any visible signs of activity against both the species of fungi C. albicans and A. fumigatus.

\section{References}

Arulmurugan, S., Kavitha, P.H., and Venkatraman, R.P. 2010. Biological activities of Schiff base and its complexes: a review. Rasayan. J .Chem. 3, 385-410.

Brogden, R.N., Heel, R.C., Speight T.M., and Avery, G.S. 1979. Amoxicillin injectable: a review of its antibacterial spectrum, pharmacokinetics and therapeutic use. Drugs 18, 169-184.

British Pharmacopoeia, 2009. London: British Pharmacopoeal Commission, pp. 353-367.

Dhar, D.N., and Taploo, C.L. 1982. Schiff bases and their applications. J. Sci. Ind. Res. 41, 501-506.

El-Sooud, K.A., Al-Tarazi, Y.H., and Al-Bataineh, M.M. 2004. Comparative pharmacokinetics and bioavailability in chickens after intravenous, intramuscular and oral administration. Veter. Res. Comm. 28, 599-607.
Gordon, R.C., Regamey, C., and Kirby, W.M.M. 1972. Comparative clinical pharmacology of amoxicillin and ampicillin administered orally. Antimicrob. Agents Chemother. 1, 504-507.

Indian Pharmacopoeia, 2007. 5th ed. Ghaziabad (INDIA): Indian Pharmacopoeal Commission, pp. 100-107.

Przybylski, P., Huczyński, A., Pyta, K., Brzezinsk, I.B., and Bartl, F. 2009. Biological properties of Schiff bases and azo derivatives of phenols. Curr. Org. Chem. 13, 124-148.

Schiff, H. 1864. Mitteilungen aus dem universitats laboratorium in Pisa: Eineneue reihe organischer basen. Justus Liebigs Ann. Chem. 131, 118-119.

Tai, D.N., Thanh, N.D., Nam, P.D., and Duc, H.T. 2007. ${ }^{1} \mathrm{H}$ and ${ }^{13} \mathrm{C}$ NMR spectra of some azomethines of 5-amino2-phenylindole series, Chem. J. 45, 642-647.

United States Pharmacopoeial. 30 and National Formulary-25 The Official Compendia of Standards, Rockvile (US): United States Pharmacopoeial Commission, 2007. pp. 1402-1407.

WHO, 2013. Model List of Essential Medicines. World Health Organization. 
